Review

\title{
$X$-ray and Neutron Diffraction in the Study of Organic Crystalline Hydrates
}

\author{
Katharina Fucke and Jonathan W. Steed * \\ Department of Chemistry, University of Durham, South Road, Durham, DH1 3LE, UK; \\ E-Mail: katharina.fucke@durham.ac.uk
}

* Author to whom correspondence should be addressed; E-Mail: jon.steed@durham.ac.uk;

Tel.: +44-191-334-2085; Fax: +44-191-334-4737.

Received: 18 June 2010; in revised form: 5 July 2010 / Accepted: 6 July 2010 /

Published: 9 July 2010

\begin{abstract}
A review. Diffraction methods are a powerful tool to investigate the crystal structure of organic compounds in general and their hydrates in particular. The laboratory standard technique of single crystal X-ray diffraction gives information about the molecular conformation, packing and hydrogen bonding in the crystal structure, while powder X-ray diffraction on bulk material can trace hydration/dehydration processes and phase transitions under non-ambient conditions. Neutron diffraction is a valuable complementary technique to X-ray diffraction and gives highly accurate hydrogen atom positions due to the interaction of the radiation with the atomic nuclei. Although not yet often applied to organic hydrates, neutron single crystal and neutron powder diffraction give precise structural data on hydrogen bonding networks which will help explain why hydrates form in the first place.
\end{abstract}

Keywords: hydrate; single crystal diffraction; powder diffraction; neutrons; X-ray

\section{Introduction}

The structure of water and the way it self-assembles and interacts with dissolved solutes and with hydrophobic surfaces continues to be highly topical [1-5]. Studies on water were ranked by Science as one of their top 10 breakthroughs of 2004 [6], and water structure in liquid as well as in the solid-state is the topic of more and more fundamental studies [7-8]. The incorporation of water molecules into the 
crystal lattice, i.e., hydrate formation, is not only of academic interest but has application or significance in a variety of contexts in the chemical industry, since this phenomenon is well known for a plethora of different chemical substances. In the pharmaceutical sector in particular, the formation of hydrates may pose severe problems during manufacturing and influence the solubility and thus the bioavailability of a drug compound. Also other branches of the chemical industry are affected by hydrate formation (e.g., in pesticides). Thus it is essential to identify and characterise hydrates in an early stage of development. One of the most powerful ways of doing so is the use of diffraction methods. Moreover, diffraction data give detailed structural information that potentially allows a level of understanding, prediction and control of hydrate formation. This brief review looks at common powder and single crystal diffraction based techniques that are applied to the study of crystalline hydrates of organic compounds and gives a brief overview of some of the fascinating structural insights that both X-ray and neutron diffraction can reveal about the structure of water in a crystalline lattice.

\section{Diffraction Techniques-General Principles}

Diffraction describes the scattering of waves (e.g., electromagnetic radiation or radiation such as neutrons with wave-like characteristics) by an object. The degree to which the wave is diffracted is at a maximum when the characteristic size of the object is of the order of the wavelength of the radiation. If the radiation interacts with a regular array of obstacles, interference patterns can be observed resulting in maxima (constructive interference between diffracted waves) and minima (destructive interference) of intensity. A single crystal has a three-dimensional repeating lattice that arises from the regular spacing of atoms or molecules within the sample, and thus gives a diffraction pattern of distinct spots (Laue spots, Figure 1a). The relative location of these spots gives the crystal symmetry (encompassed in the crystallographic space group) and the dimensions of the unit cell (the basic 'building block' of the crystal) according to Bragg's law (Equation 1). Here $n$ is an integer, $\lambda$ is the wavelength of the radiation, $\theta$ is the angle through which the radiation is diffracted and $d$ represents the characteristic spacing distance of the lattice.

$$
n \lambda=2 d \sin \theta
$$

Figure 1. Diffraction from (a) an oriented single crystal, (b) a collection of four crystals and (c) from a polycrystalline material, with (d) the cross-section of the Debye-Scherrer rings ([9]—reproduced by permission of The Royal Society of Chemistry).

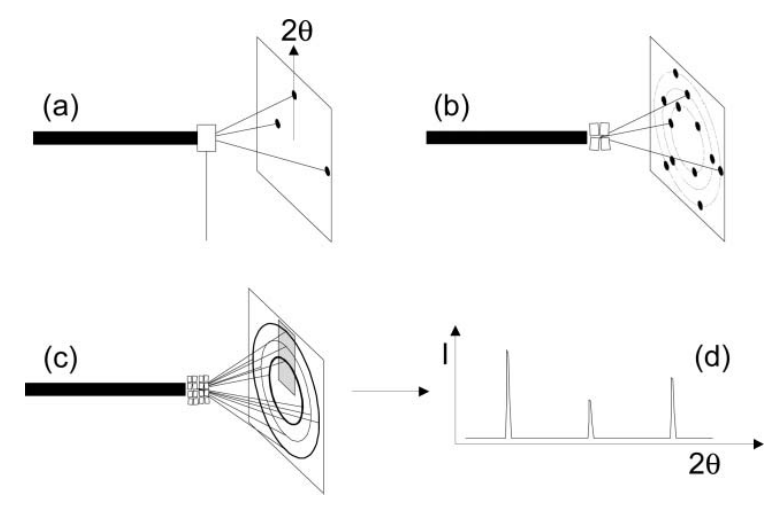


The intensity of the diffraction maxima holds information about the contents of the unit cell including the molecular structure and electron density distribution (or in the case of neutron diffraction, nuclear density distribution).

For powder diffraction the same principles hold true. Powder samples usually consist of a multitude of micron-sized single crystals with a random or statistical orientation. Thus the distinct Laue spots observed for a single crystal are spread out into cones of diffraction giving rise to rings (Debye-Scherrer rings, Figure $1 \mathrm{~b}$ and $1 \mathrm{c}$ ) on a two dimensional detector. A cut through these rings gives the one dimensional powder diffractogram (Figure 1d). As in the case of the Laue spots, the position of the Debye-Scherrer rings gives information about the unit cell and its symmetry, while the intensity of the reflections is due to the contents of the unit cell.

In order to diffract from a crystal, high energy radiation such as X-rays from a molybdenum (wavelength $0.7107 \AA$ ) or copper (wavelength $1.5451 \AA$ ) radiation source is used on the laboratory scale. Additional possibilities are high brilliance rotating anode diffractometers or the use of synchrotron radiation, especially for problematic or very small crystals. The X-rays are diffracted by the electrons of the atoms, thus the more electrons an atom has, the stronger it diffracts. While an advantage for locating heavy atoms, this characteristic poses major problems in the determination of accurate hydrogen atom positions (especially in the presence of heavy atoms that dominate the diffraction pattern) as well as in distinguishing atoms of different elements that are close together in the periodic table (e.g., carbon and nitrogen). Moreover, in hydrogen the electron is not generally located near the atomic nucleus, but instead is generally involved in a covalent bond to a neighbouring atom. As a result the positions of hydrogen atoms located using X-rays are typically systematically displaced towards the atom to which the hydrogen is bound and consequently bond lengths from hydrogen to other elements measured by X-ray methods are consistently shorter than their 'true' values even in a high precision experiment. The problem worsens at higher temperature because the bonded electrons are subject to increased thermal motion (libration) perpendicular to the bond resulting in a shortened average position apparent from the X-ray data (Figure 2). The thermal motion of strongly hydrogen bonded hydrogen atoms has been accurately studied by neutron diffraction [10]. However, since X-rays sources are relatively cheap and readily available most solid-state laboratories possess an $\mathrm{X}$-ray diffractometer and this method is the most widely used diffraction technique. If really accurate hydrogen atom positions are required an alternative is neutron diffraction, described below.

Figure 2. Librational apparent shortening of bonds to hydrogen (reproduced with permission from [11]).

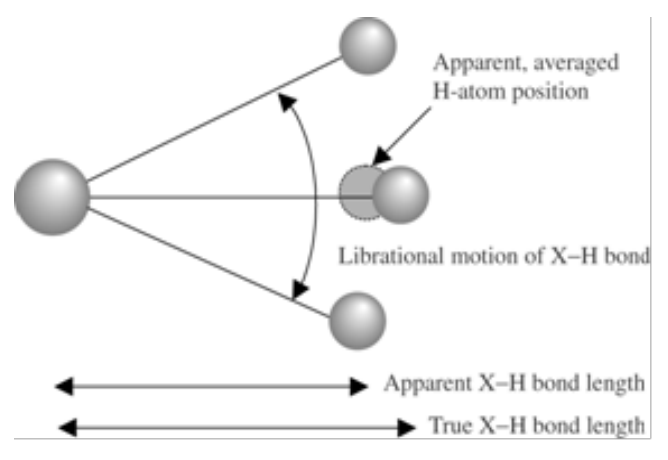




\section{Single Crystal X-ray Structures of Hydrates}

Single crystal X-ray diffraction, due to its accuracy and its informative value, is one of the most important tools in chemical research and in industry. A model of the molecular structure and connectivity can usually be relatively easily derived from the diffraction pattern by Fourier transform and is normally the final proof of identity for a newly prepared compound, a specific crystal form or an extracted substance. Due to the precision of the method (standard errors on bond lengths are of the order of $0.01 \AA$, or often much less), the structural models can be interpreted in terms of crystal packing and intermolecular interactions.

Concerning the packing, most crystal forms are distinctly different. However, isostructural crystals exist, which show the same arrangement of molecules (i.e., packing) although the unit cell dimensions and the chemical composition can differ. In the special case of isomorphous crystals, the different forms are isostructural and also have the same space group and unit cell dimensions but different chemical composition.

The most obvious intermolecular interaction in crystals is the hydrogen bond, in which a hydrogen bond donor group, typically an $\mathrm{OH}$ or $\mathrm{NH}$ functionality, interacts with a hydrogen bond acceptor group, usually an electronegative atom with a non-bonding electron pair such as a $\mathrm{C}=\mathrm{O}$ group, although interactions to alternative acceptors such as aromatic rings are also known [12]. In hydrates, the hydrogen bond lengths range from 1.5 to $2.7 \AA$ with an angle ranging between 160 and $180^{\circ}$ [13]. Although the strongest hydrogen bond has an angle of $180^{\circ}$, i.e., donor, hydrogen and acceptor are on a straight line, smaller angles are statistically favoured due to the increasing number of possible binding orientations (conic factor). Thus the angle distribution shows a maximum at about $160^{\circ}$, but a statistical conical correction factor gives the expected maximum at $180^{\circ}$ [14].

The Cambridge Structural Database (CSD) [15] contains over 500,000 entries of organic and metal-organic crystal structures, of which 70,484 structures contain water in one form or another. A number of studies have been undertaken to evaluate this huge amount of structural data in order to gain insight in hydrate formation and develop firm rules for the prediction of their structure and occurrence. Desiraju [16] has investigated a test-set of 411 hydrate crystal structures and found that although manifold factors play a role in hydrate formation, this process is more likely to happen when the host molecule shows an imbalance between the number of hydrogen bond donor and acceptor groups. Gorbitz and Hersleth [17] have discussed the increase in recent years of occurrence of solvated crystal forms, including hydrates and mixed solvates (i.e., water and an organic solvent), and conclude that this increase is due to the increasing complexity of modern molecules and their reduced packing efficiency. Infantes and Motherwell [18] investigated 1516 organic hydrate structures of the CSD with a view to classifying water clusters. They found that the majority of structures (61\%) have distinct finite chain motifs, and of these usually only two water molecules hydrogen bond to each other, representing the whole chain. Infinite chain motifs at almost $20 \%$ of the sample are the second most common pattern. The authors also found that a high percentage of the chain, tape and layer motifs are oriented along the shortest cell axis. There has been an increasing recent fashion towards the interpretation of hydrate structures in terms of new motifs and connectivities in the water clusters, however Mascal et al. [19] have shown that such reports have to be taken with a pinch of salt, as a considerable number of purportedly "new” clusters are in fact already known. 
Another CSD evaluation by Infantes et al. [18] deals with the probability of hydrate formation if the host molecule has certain chemical groups. As anticipated, hydrate formation is most frequent when charged groups are present. But also carbonyl $(\mathrm{C}=\mathrm{O})$, ether $(\mathrm{C}-\mathrm{O}-\mathrm{C})$ and hydroxyl $(\mathrm{OH})$ groups increase the probability of water incorporation. From a test set containing 5,605 hydrate crystal structures, the majority incorporate the water molecules in a threefold hydrogen bonding pattern, in good agreement to earlier reports [13,20]. From a test set of 6,558 hydrate structures, Infantes et al. [21] conclude that hydrate formation does not depend on the hydrogen bond donor/acceptor ratio but rather on the sum of all donor and acceptor groups in the host molecule and on the total polar surface area. Haynes et al. have investigated more specifically hydrate formation in pharmaceutical salts [22,23]. These authors found that the percentage of hydrate formation in this subgroup of the CSD is generally higher than seen in the database as a whole [22]. Additionally, doubly charged counter ions increase the possibility of water incorporation even more, with the proportion forming hydrates greater than $50 \%$. The only triply charged counter ion $\left(\mathrm{PO}_{4}{ }^{3-}\right)$ studied forms hydrates as the only known crystal forms. In the $\mathrm{NH}^{+}$-containing subgroup of pharmaceutical salts, hydrate formation was found to decrease with increasing ion size in the halide series, while secondary and tertiary amine salts tend to form fewer hydrates [23]. A hypothesis has been put forward by Steed and coworkers that allows some qualitative prediction of the likelihood of hydrate formation in salts based on anion-cation spacing and charge arguments [24]. Overall, these studies of the CSD show some strong trends in hydrate formation, which subsequently can be used as input for $a b$ initio crystal structure prediction [25].

Hydrates can be generally classified into two categories, the stoichiometric ones with a fixed ratio of water to host compound, and the non-stoichiometric ones, in which the water contents can reversibly change due to changing outer conditions without a major change of the crystal structure. Although it is difficult to determine from the crystal structure whether a hydrated crystal form is stoichiometric or non-stoichiometric, there are certain structural characteristics that indicate a tendency to belong to one group or the other [26]. Water molecules can be situated in isolated sites as a component of the molecular network and form hydrogen bonds to the host and to other, symmetrically independent water molecules. This characteristic is mainly found in stoichiometric hydrates. It can be assumed for these compounds that the water cannot be easily removed from the positions it occupies and that its removal results in the destruction of the crystal architecture. One example of such a hydrate is the monoclinic 5-azauracil monohydrate (Figure 3) [27]. The water forms hydrogen bonds only to the host molecules, acting twice as donor and once as acceptor, while the next crystal plane is shifted so that the water molecules are embedded in a matrix of host molecules.

The water can also be located in discrete pockets forming clusters of different size depending on the number of water molecules involved. One example is a dipyridyl bis(urea) compound, which incorporates one molecule of water per 6 molecules of host [28]. The water molecules are located in discrete cavities enclosed by host molecules. Another example is reported by Byrne et al. [29] in which a compartmental coordination polymer forms a Borromean weave network. This structure incorporates discrete clusters of seven water molecules in pockets, where they form hydrogen bonds to each other as well as to the host molecules (Figure 4). As reported in both cases, the water is bound extremely strongly and not released before the decomposition of the whole structure as shown by thermogravimetric studies. Another example of this inclusion type has been reported by 
Barbour et al. [30] who investigated the crystal structure of a supramolecular cage-like complex and found larger voids due to the packing in the crystal structure. These voids are filled with ten water molecules arranged in the same cluster as can be found in cubic ice $I_{C}$ [31].

Figure 3. Crystal packing in 5-azauracil monohydrate in which the water molecules occupy isolated sites [27].

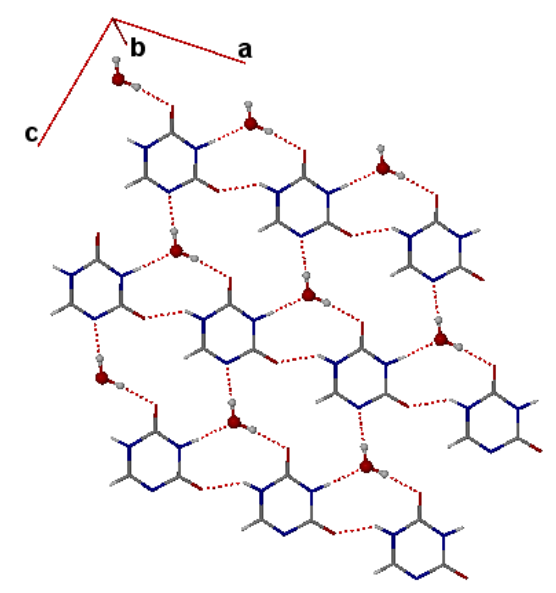

Figure 4. Discrete water heptamer in a Borromean structure [29].

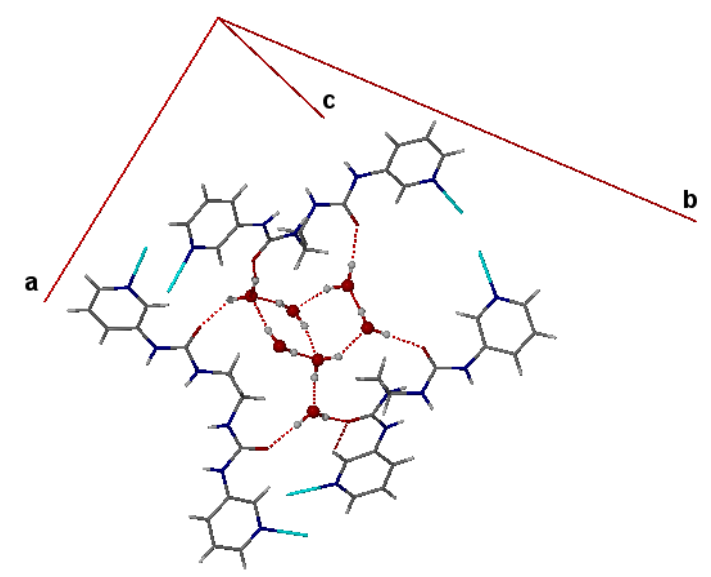

The channel hydrates present another large group of structurally related hydrate crystal forms. They incorporate water in channels or networks of channels throughout the crystal. Depending on the extent of hydrogen bonding and the size of the channels, these hydrates can be either stoichiometric or non-stoichiometric. Thymine forms a monohydrate [33] with the water molecules located in such channels along the $c$-axis (Figure 5a). Perrier and Byrn investigated the dehydration behaviour of thymine monohydrate and found that the dehydration proceeds along these channels [34]. Since this process is accompanied by pseudomorphosis, i.e., the destruction of the mother crystal into a multi-crystalline powder of the same shape, indicated by the clear single crystal turning opaque, the loss of water is connected with a major change in structure as is characteristic for stoichiometric hydrates. Caffeine 0.8 hydrate [35-37] and theophylline monohydrate [38,39] form channels in their structure as well. Both materials are isostructural and stoichiometric, while the water release behaviour from the channels differs significantly [40-42]. Another example of a channel hydrate is given in the 
co-crystal of pamoic acid and piperazine, which forms a trihydrate [32]. Interestingly, two of the water molecules are located in helical channels in the structure (Figure 5b) surrounded by three host helices. The remaining water molecule links the host helices and is situated in isolated sites. The helical water can easily leave the crystal lattice through the channels without the overall structure to collapse, as the authors show by drying and rehydrating. This is a characteristic feature of a non-stoichiometric hydrate. Larger channels are more likely to be a structural feature of non-stoichiometric hydrates, as the water can diffuse more easily through these channels without the general structure changing. Cephalexin dihydrate [43] shows such large channels along the $a$-axis. The water molecules are mostly disordered indicating already the higher mobility than in caffeine or theophylline. Stephenson et al. [44] proved that this hydrate behaves as a non-stoichiometric material at least for a water content of one to two molecules of water per molecule of cephalexin. Saha and Nangia [45] have described another case of channel hydrate formed by dibromophloroglucinol. Interestingly, the water coordinates in the channels in a hexamer boat type structure which can also be found in hexagonal ice $\mathrm{I}_{\mathrm{h}}$. The authors describe the dehydration as reversible while it cannot be said whether the hydrate is stoichiometric or non-stoichiometric since the rehydration was carried out in a water saturated atmosphere- a condition in which both kinds of hydrates can be rehydrated.

Figure 5. (a) Stacking of thymine monohydrate with the water channels along $c$ and (b) helical structure of the pamoic acid/piperazine co-crystal trihydrate (reprinted with permission from [32], Copyright 2009 American Chemical Society).
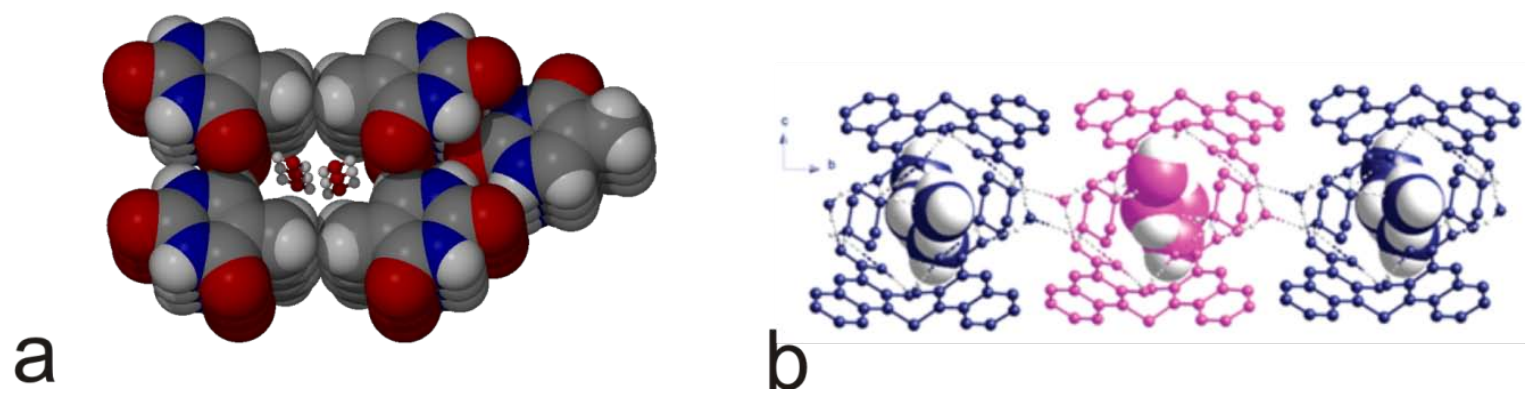

Figure 6. Discrete water layer in uric acid dihydrate.

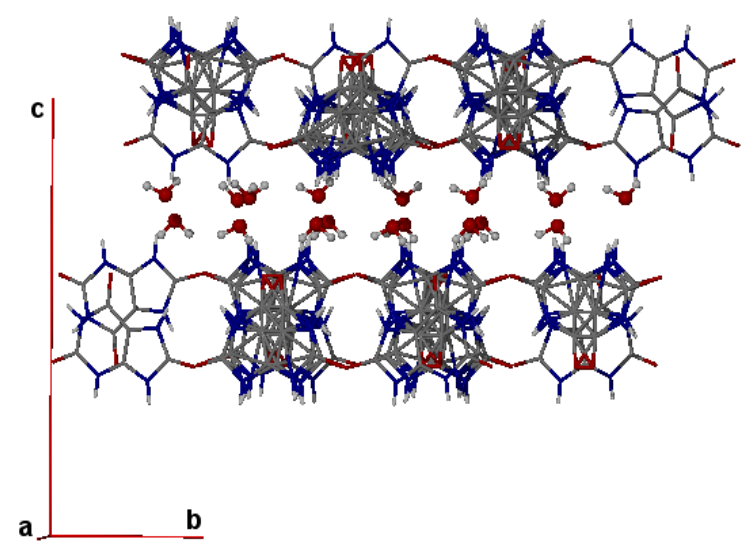


Some hydrates contain the water in discrete layers in the crystal structure, as shown by the example of uric acid dihydrate (Figure 6) [46-48]. Although this feature is closely connected to higher guest mobility and thus could be assumed to be most common in non-stoichiometric hydrates, uric acid dihydrate in fact represents a stoichiometric hydrate which undergoes strong pseudomorphosis when dried. 2,2'-dipyridylamine dihydrate also incorporates water as highly hydrogen-bonded network in layers between layers of the host molecules [49]. Although no dehydration studies has been carried out so far, it can be assumed from the extent of hydrogen bonding that this crystal form is also a stoichiometric hydrate.

Water molecules can be strongly coordinated to small metal ions such as $\mathrm{Na}^{+}, \mathrm{K}^{+}$or $\mathrm{Mg}^{2+}$. For transition metals, this interaction is particularly strong. In fact, $5.7 \%$ of the metal-organic structures listed in the CSD [15] in 2000 include water as ligand to the metal ion, more than any other solvent molecule [51]. These aquo complexes reveal significant stability with water loss occurring well above the boiling point of liquid water, probably as a result of ion dipole interactions with the metal centre. Examples of such highly stable coordination complexes are the pigments yellow 183 $\left(\mathrm{Ca}\left(\mathrm{C}_{16} \mathrm{H}_{10} \mathrm{C}_{12} \mathrm{~N}_{4} \mathrm{O}_{7} \mathrm{~S}_{2}\right)\right.$, Figure 7) and yellow $191\left(\mathrm{Ca}\left(\mathrm{C}_{17} \mathrm{H}_{13} \mathrm{ClN}_{4} \mathrm{O}_{7} \mathrm{~S}_{2}\right)\right)$ [50]. The $\alpha$ forms of both pigments contains a single water molecule per formula unit, while the $\beta$ forms have three water molecules. In all crystal forms, the water is coordinated to the calcium ions (green nodes in Figure 7) and additionally hydrogen bonded to the host molecules. The $\alpha$ forms are described to be stable up to $523 \mathrm{~K}$ [50,52], well above the boiling point of pure water. Interestingly, this dehydration temperature is $100 \mathrm{~K}$ higher than that of the related inorganic gypsum $\left(\mathrm{CaSO}_{4} \cdot 2 \mathrm{H}_{2} \mathrm{O}\right)$ [53]. The $\beta$ forms are less stable and transform to the monohydrates at lower temperatures.

Figure 7. Pigment yellow 183 (a) $\alpha$ form and (b) $\beta$ form. The green nodes represent $\mathrm{Ca}^{2+}$ ions [50].
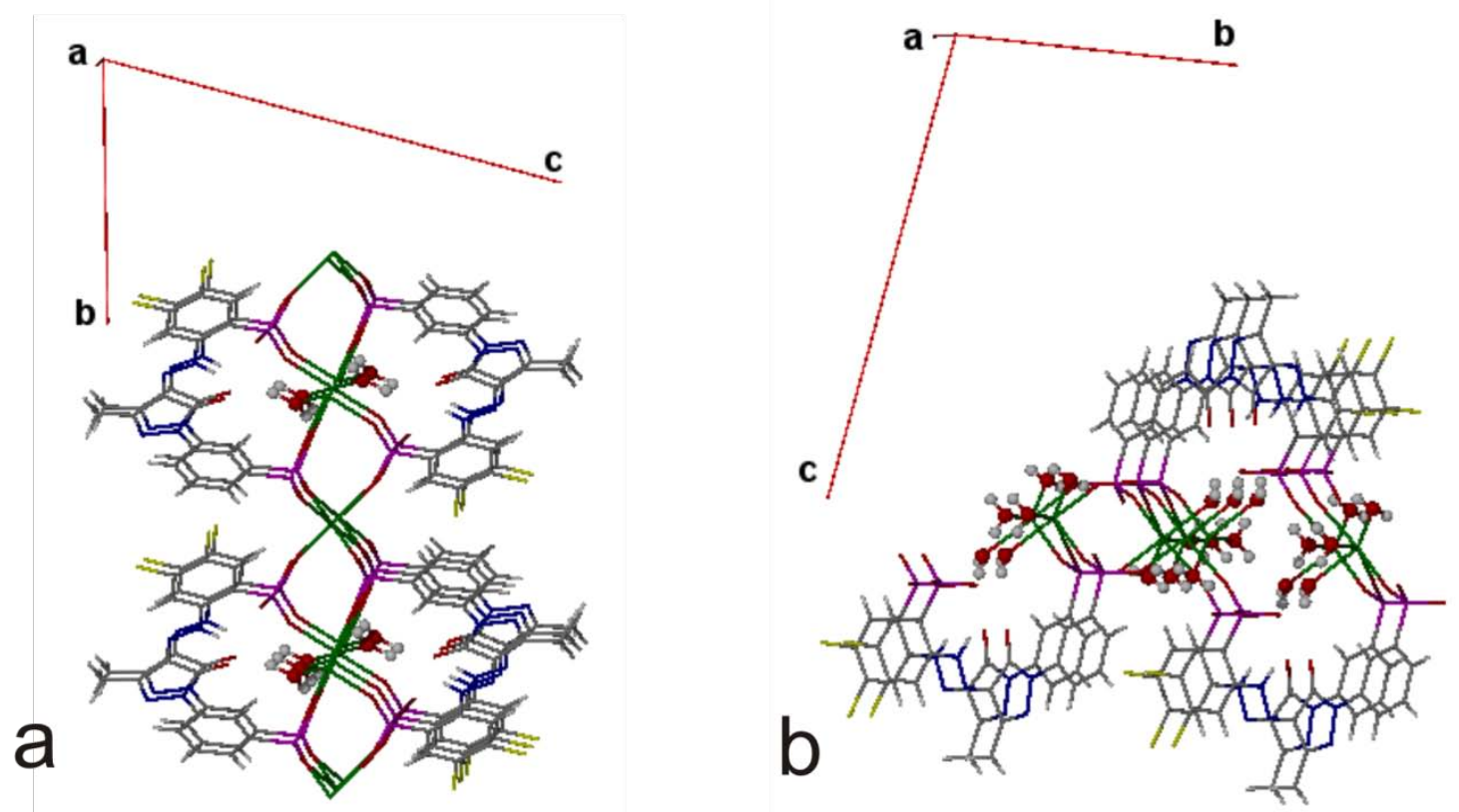

It is not always true that related compounds crystallise as related hydrates, as shown by Oxtoby et al. [54] The authors investigated a set of four closely related 4,4-dihydroquinoxaline-2,3-diones in 
order to understand water cluster formation, which resulted in the observation of zero-, one- and two-dimensional arrays of water. The authors concluded that in the formation of these hydrated crystal forms not only the strong hydrogen bonds are involved but indeed at number of competing and relatively weak interactions control the formation of specific arrays.

Overall, these examples show that although some properties of hydrates might be obvious in the crystal structure, diffraction methods alone are not sufficient to characterise the crystal forms. Normally, a wide range of analytical tools is employed, e.g., thermomicroscopy, differential scanning calorimetry, thermogravimetry and dynamic vapour sorption, to fully understand the behaviour and stability of hydrated crystal forms.

\section{Powder X-ray Diffraction}

Many compounds, especially pharmaceuticals and larger bulkier molecules, tend not to grow readily as single crystals of sufficient size to determine the crystal structure. In these cases powder X-ray diffraction (PXRD) is a more powerful and versatile tool to identify and characterise crystal forms. Most solid-state studies now present the PXRD patterns as a characteristic fingerprint of a particular crystalline phase. The example of piroxicam shows the value of this technique, since all four different crystal forms are unambiguously identifiable from their PXRD patterns (Figure 8) [55]. All the information needed to calculate the appearance of a PXRD pattern is included within single crystal data and comparison of powder patterns calculated from single crystal structures with powder patterns of the bulk material gives useful information, such as whether the single crystal structure is representative of the bulk or if the sample shows preferred orientation, i.e., whether the crystals in the powder are arranged in a preferred manner. For example plates mainly pack with contact to neighbouring crystals on the largest face, while needles will often align parallel to each other. This kind of preferred orientation effects can result in marked intensity variations in the powder pattern. In addition, care must be taken in comparing PXRD data which are generally obtained at room temperature, with powder patterns calculated from single crystal data, which are often obtained at low temperature (frequently 90-150 K) with consequent shrinking of the unit cell parameters and hence differences in peak position. In some cases, particularly for relatively rigid molecules it is readily possible to determine the full crystal and molecular structure from powder data. This methodology which results in three-dimensional information from one-dimensional PXRD data is not within the scope of this review but is covered extensively elsewhere [56-58]. However, if the powder pattern can be indexed and the asymmetric unit does not present major difficulties (large size, more than one molecule, molecules on special positions like mirror planes, rotation axes or inversion centres), the crystal structure can be relatively quickly solved and refined from powder data. One successful example is risedronate sodium anhydrate $\left(\mathrm{C}_{7} \mathrm{H}_{10} \mathrm{NO}_{7} \mathrm{P}_{2} \mathrm{Na}\right)$ [59]. The complexity of the problem is significantly increased for hydrates since the presence of the water molecule(s) significantly increases the number of degrees of freedom of the system. 
Figure 8. PXRD patterns of piroxicam form I (1), form II (2), form III (3) and the monohydrate (4) (reprinted from [55], Copyright 2003, with permission from Elsevier).

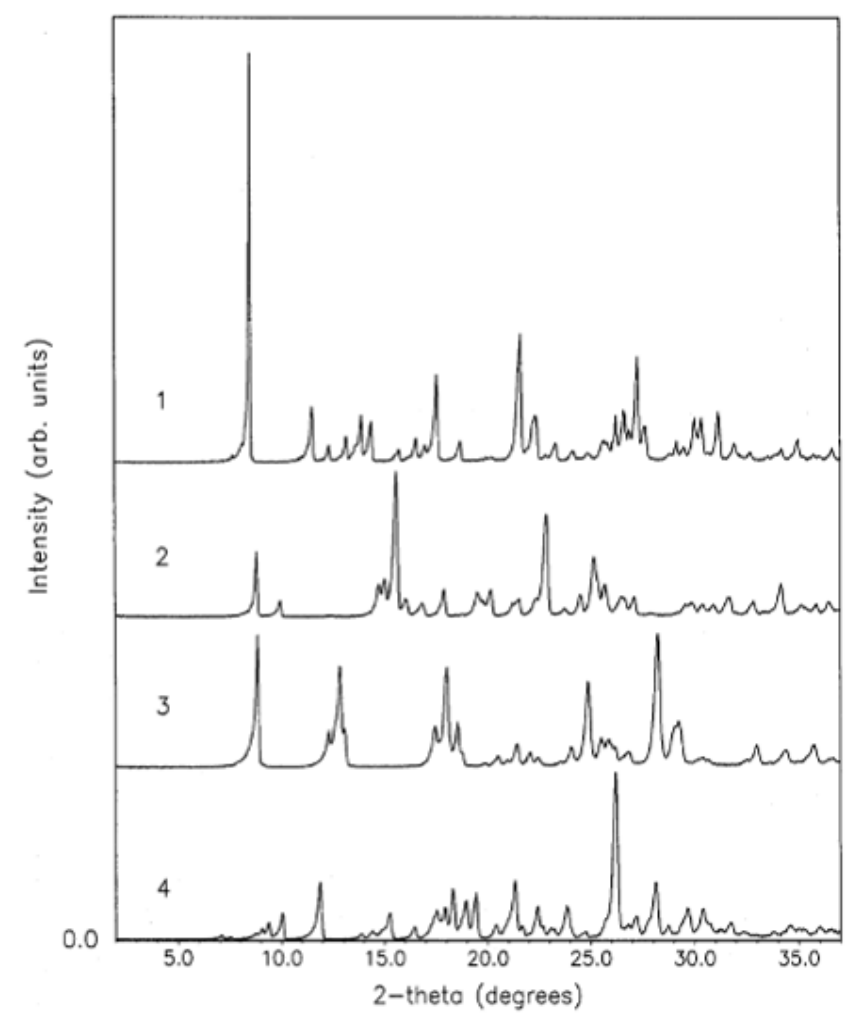

Structural details from PXRD are not as precise as single crystal data and it is not generally possible to refine hydrogen atom positions. However, this method might sometimes present the only possibility to obtain structural details at all in cases where suitable single crystals are not available. On the other hand, it is not always necessary to collect a full data set for structure refinement, e.g., when the change in the unit cell upon drying is determined, it normally is sufficient to obtain the structural model at one point and refine the cell parameters from powder data [9].

Another application of PXRD is measurement at non-ambient conditions. Since not all phase transitions follow a single-crystal-to-single-crystal mechanism [60-61], investigating such events with single crystal X-ray diffraction proves difficult at best. PXRD is measured in the bulk with randomised orientation and thus the conditions can be varied widely without loss of applicability of the method. For any crystal form in general, and the hydration/dehydration processes of hydrates in particular, processes can be studied either by varying the temperature (variable temperature or temperature controlled PXRD) or the humidity (humidity controlled PXRD). Temperature resolved PXRD is more commonly used [62], e.g., for the characterisation of berberine hydrochloride hydrates [63]. The authors show that the dihydrate dehydrates to the anhydrate in the range from 60 to $70{ }^{\circ} \mathrm{C}$, while the tetrahydrate dehydrates to the dihydrate between 25 and $30{ }^{\circ} \mathrm{C}$. These data correlate well with the thermal analysis but gives additionally proof of the crystal form. Another example is the thermal dehydration of nedocromil magnesium pentahydrate (Figure 9) [64], which shows the slight differences in dehydration behaviour if heated in an open system, where the water can readily leave the 
direct environment of the sample, and a closed system, in which the surrounding sample's atmosphere is saturated with water and the dehydration thus hindered.

Figure 9. Nedocromil magnesium pentahydrate undergoing the complete transition to the dehydrated form while heated in (a) an open system and (b) a closed system (reprinted from [64], Copyright 2001, with permission from Elsevier).
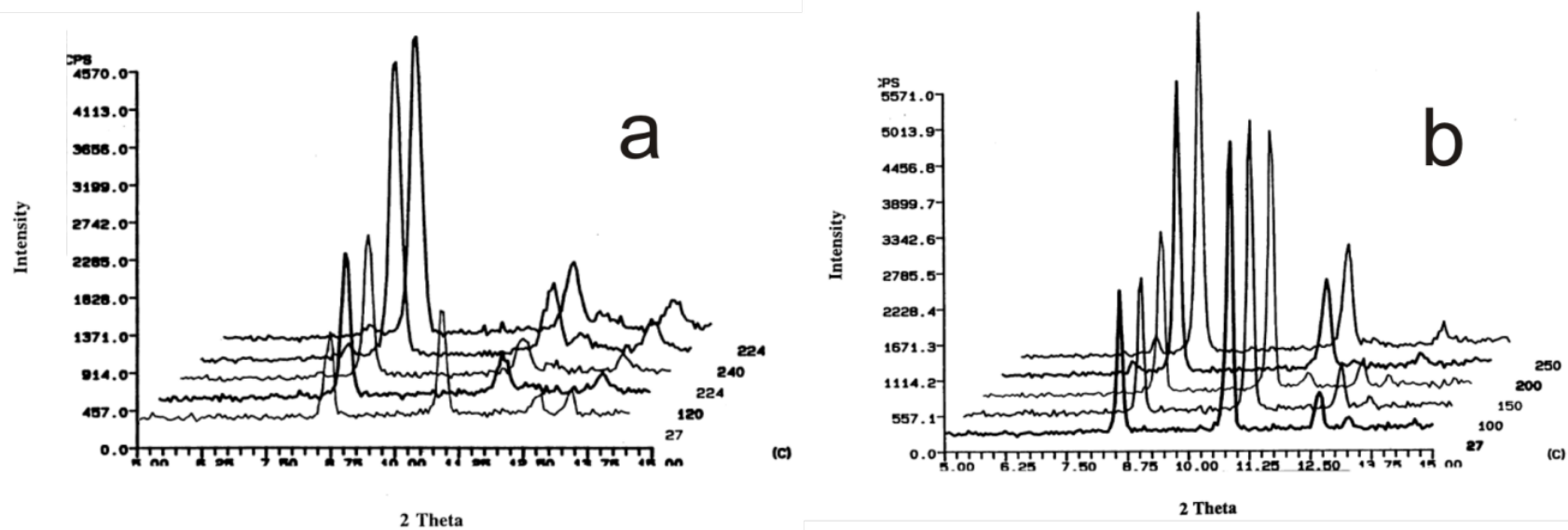

Paracetamol shows a humidity triggered phase transition of its solution grown form II to the stable form I (Figure 10) [65]. Form II is the thermodynamically metastable polymorph at room temperature but is of interest to the pharmaceutical industry to process, since this crystal form can be directly compacted into tablets, whereas form I has to be granulated with excipients in a separate step before compaction. However, the authors found that the solution grown crystals contain a minor amount of residual solvent which at elevated humidities triggers the transformation of the metastable form II into the stable form I.

Figure 10. Time-resolved PXRD patterns of orthorhombic paracetamol (form II) at 90\% relative humidity, recorded sequentially every $60 \mathrm{~min}$ (first pattern in the front).

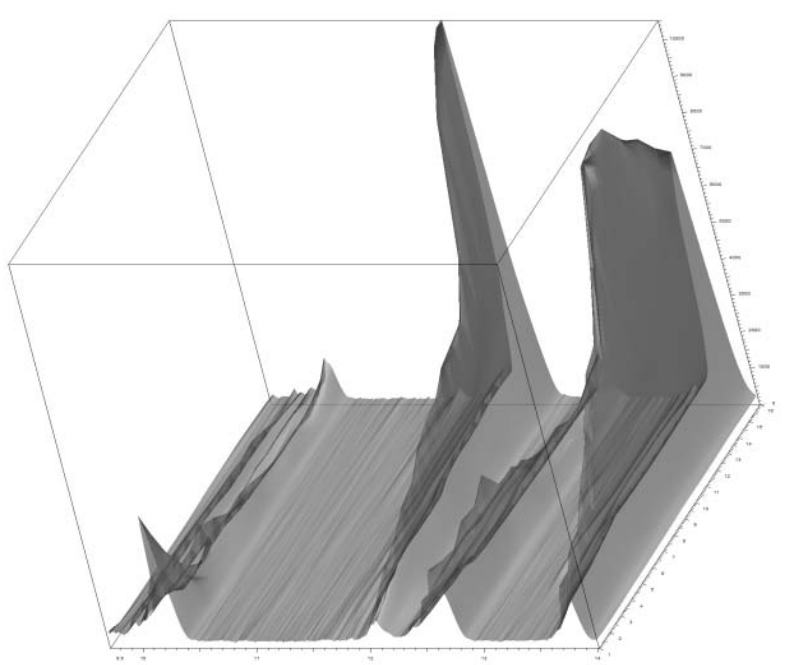


One recent development has shown that the crystal structure of an unusual hydrate material derived from a hydrogel of melamine and uric acid can be derived by ab initio crystal structure calculation and comparison of the resulting calculated PXRD pattern with the broad PXRD pattern obtainable on such poorly crystalline xerogel materials [66].

\section{Neutron Diffraction}

An alternative radiation for diffraction experiments is neutron radiation. Due to the particle/wave dualism, neutrons can be diffracted like X-rays, and depending on their energy, the wavelength can be tuned to match the problem at hand. Neutrons do not interact with the electron shell but with the nucleus of atoms, more specifically with its spin, so that the scattering power does not linearly increase with the atom number, unlike X-rays. In fact, the scattering lengths of elements seem to follow an arbitrary course, and even different isotopes of the same element can have completely different scattering lengths (e.g., hydrogen has a coherent scattering length of $-3.742 \mathrm{fm}$, while that of deuterium is $6.674 \mathrm{fm}$ ). Therefore, neutron scattering is a well recognised method for the determination of accurate hydrogen positions [67]. Neutrons are generated at a limited number of installations by either nuclear reactors (such as the Institute Laue Langevin in Grenoble, France) or at a pulsed spallation source such as ISIS in the UK and as a result measurement time on neutron diffractometers is at a premium. However, because neutrons interact with the atomic nucleus rather than the electrons they give a more accurate measure of hydrogen atom position. Moreover, since neutron scattering does not increase as a function of atomic mass, hydrogen atoms (and particularly the heavy hydrogen isotope deuterium) contribute significantly to a neutron diffraction pattern. Aside from the cost, however, disadvantages of neutron diffraction include the generally weak diffraction of neutrons by crystals which means that very large samples (upwards of $1 \mathrm{~mm}^{3}$ ) are needed in comparison to samples for X-ray work where single crystals as small as $2 \times 10^{-4} \mathrm{~mm}^{3}$ are sometimes adequate.

Like X-ray diffraction, neutron diffraction can be executed on single crystals as well as on powders. Since it is not always possible to obtain sufficiently large single crystals, neutron single crystal diffraction is still a speciality method and not used routinely. Additionally, although feasible, without an initial X-ray model it is rather hard to determine crystal structures from the neutron diffraction, due to the positive and negative nature of the scattering lengths, which further complicates the assignment of phases. However, once obtained, neutron diffraction data give valuable information about absolute atomic positions and bond lengths especially involving hydrogen [68-71].

One technique to minimise the size of crystal needed is to use Laue diffraction which involves a range of wavelengths (typically ca. 1-5 $\AA$ ) in order to satisfy the Bragg diffraction condition (Equation 1) for many reflections simultaneously. This approach contrasts to the more typical monochromatic approach. This method is implemented for the LADI and VIVALDI diffractometers at ILL and KOALA in the Bragg Institute in Australia, for example. These facilities also have the advantage of relatively routine access to very low temperatures down to $c a$. $4 \mathrm{~K}$, minimizing atomic motion and further enhancing precision. Turner et al. [72] have used the Laue technique to study a metal-organic structure which incorporates water in two different environments. Two of the four incorporated water molecules are coordinated to the metal ion (cobalt(II)), while the remaining two 
water molecules hydrogen bond to the ligands. The authors found that the hydrogen bonds involving the metal coordinated water molecules are shorter (hydrogen to acceptor atom distance) but according to energy calculations less strong than the ones between the free water and the ligand molecules. Ferraris and Franchini-Angela [73] have studied the environment of water in 41 neutron structures of inorganic hydrates and can classify these structures into three classes of water-cation interactions. Although challenging, neutron structures for large biological molecules like proteins have been reported. Bouquiere et al. [74,75] determined the neutron structure of vitamin $\mathrm{B}_{12}$ coenzyme measured at $15 \mathrm{~K}$ and determined additionally the water structure in the binding pocket. The authors show that the water is incorporated in two distinctly different domains, a channel with statically disordered water molecules and adjacent to that a pocket with highly ordered solvent. Bilton et al. report the crystal structure of a gem-alkynol compound $\left(\mathrm{H}_{10} \mathrm{H}_{4} \mathrm{Br}_{4} \mathrm{O}_{2} \cdot 2 \mathrm{H}_{2} \mathrm{O}\right)$, for which a complex hydrogen bonding scheme could be determined by neutron diffraction [76]. The incorporated water shows in fact the uncommon feature of accepting two hydrogen bonds while both water hydrogen atoms are bifurcated, both donating to an oxygen and a bromine atom. Unfortunately, single crystal neutron diffraction is still mainly applied to inorganic structures or magnetic and superconducting materials rather than small organic molecules and their hydrates, while this technique would give especially in the case of hydrates valuable and accurate information about the water molecules.

Neutron powder diffraction is another possibility to obtain accurate structural data if no sufficiently sized single crystal can be obtained. The theory of this method is the same as for powder X-ray diffraction, but samples containing a large proportion of hydrogen pose the problem of very high backgrounds due to the incoherent scattering of hydrogen (scattering correlated to movement of the atoms and carrying no structural information). Thus, the sample should be deuterated if possible. Like neutron single crystal diffraction, neutron powder diffraction is usually employed to refine a structural model obtained from X-ray experiments, or more specifically for this method, to follow certain reactions under non-ambient conditions. However, the structure can be directly solved from powder data, as shown by Krebs et al. [77]. Although neutron powder diffraction data were only recorded to demonstrate a neutron diffraction inert cryoprotectant, the collected data of hydrogenated L-(-)-ephedrine hemihydrate could be solved and refined directly without an X-ray model. However, most applications of neutron powder diffraction are carried out to follow changes in the crystal structure [78], e.g., when atoms and molecules are absorbed [79,80] or the crystal structure changes due to strain [81,82]. Neutron powder data retain the advantage of the strong scattering by particular nuclei, especially deuterium and of course does not suffer from the sporadic availability of the very large single crystals needed for single crystal neutron work. However, significant quantities of deuterated powder are needed to overcome the intrinsically low flux of neutron sources.

\section{Conclusion and Perspective}

Hydrate formation, along with co-crystals in general [83], is a highly topical field of research with important practical applications in pharmaceutical formulation as well as wide-ranging theoretical interest. Using diffraction methods, hydrated crystal forms can be unambiguously identified and characterised. Moreover, single crystal diffraction methods give information about the structural features of hydrates and exquisite detail concerning their hydrogen bonding schemes. Neutron 
diffraction is a valuable tool especially in this area, since it allows determining precise atomic coordinates of hydrogen atoms, a task which is normally not feasible with standard X-ray methods. Powder diffraction experiments can be conducted when no single crystals are available. The layout of the experiment additionally allows time resolved solid state processes such as dehydration and phase transitions to be studied both qualitatively and quantitatively from a kinetic standpoint. Although sparsely applied, neutron powder diffraction experiments are applicable in cases where hydrogen atom positions are of particular importance, but the high background from the hydrogen atoms prohibits this use in most cases. Overall, diffraction methods prove to be an essential tool to understand hydrated crystal structures, the causes of their formation and their stability.

\section{Acknowledgement}

We thank the EPSRC for funding.

\section{References}

1. Yoshizawa, M.; Kusukawa, T.; Kawano, M.; Ohhara, T.; Tanaka, I.; Kurihara, K.; Niimura, N.; Fujita, M. Endohedral clusterization of ten water molecules into a "molecular ice" within the hydrophobic pocket of a self-assembled cage. J. Am. Chem. Soc. 2005, 127, 2798-2799.

2. Henry, M.; Bogge, H.; Diemann, E.; Muller, A. Chameleon water: assemblies confined in nanocapsules. J. Mol. Liq. 2005, 118, 155-162.

3. Papoian, G.A.; Ulander, J.; Eastwood, M.P.; Luthey-Schulten, Z.; Wolynes, P.G. Water in protein structure prediction. Proc. Natl. Acad. Sci. USA 2004, 101, 3352-3357.

4. Henry, M.; Taulelle, F.; Loiseau, T.; Beitone, L.; Ferey, G. Is water templating nanoporous materials? Chem. Eur. J. 2004, 10, 1366-1372.

5. Koop, T. Homogeneous ice nucleation in water and aqueous solutions. Z. Phys. Chemie-Int. J. Res. Phys. Chem. Chem. Phys. 2004, 218, 1231-1258.

6. Breakthrough of the Year-The Runners-Up. Science 2004, 306, 2013-2017.

7. Soper, A.K.; Teixeira, J.; Head-Gordon, T. Is ambient water inhomogeneous on the nanometer-length scale? Proc. Natl. Acad. Sci. USA 2010, 107, E44.

8. Salzmann, C.G.; Radaelli, P.G.; Mayer, E.; Finney, J.L. Ice XV: a new thermodynamically stable phase of water ice. Phys. Rev. Lett. 2009, 103, 105701.

9. Evans, J.S.O.; Radosavljevic Evans, I. Beyond classical applications of powder diffraction. Chem. Soc. Rev. 2004, 33, 539-547.

10. Wilson, C.C.; Shankland, N.; Florence, A.J. A single-crystal neutron diffraction study of the temperature dependence of hydrogen-atom disorder in benzoic acid dimers. J. Chem. Soc.Faraday Trans. 1996, 92, 5051-5057.

11. Steed, J.W.; Atwood, J.L. Supramolecular Chemistry, 2nd ed.; Wiley: Chichester, UK, 2009; p. 449.

12. Snowden, T.S.; Bisson, A.P.; Anslyn, E.V. A comparison of NH- $\pi$ versus lone pair hydrogen bonding effects on carbon acid $\mathrm{p} K_{\mathrm{a}}$ shifts. J. Am. Chem. Soc. 1999, 121, 6324-6325.

13. Gillon, A.L.; Feeder, N.; Davey, R.J.; Storey, R. Hydration in Molecular Crystals-A Cambridge Structural Database Analysis. Crys. Growth Des. 2003, 3, 663-673. 
14. Jeffrey, G.A. An Introduction to Hydrogen Bonding; Oxford University Press: New York, NY, USA, 1997; pp. 15-16.

15. Allen, F.H. The Cambridge Structural Database: a quarter of a million crystal structures and rising. Acta Crystallogr. Sect. B 2002, B58, 380-388.

16. Desiraju, G.R. Hydration in organic crystals: prediction from molecular structure. Chem. Commun. 1991, 6, 426-428.

17. Gorbitz, C.H.; Hersleth, H.-P. On the inclusion of solvent molecules in the crystal structures of organic compounds. Acta Crystallogr. Sect. B 2000, B56, 526-534.

18. Infantes, L.; Chisholm, J.; Motherwell, S. Extended motifs from water and chemical functional groups in organic molecular crystals. Cryst. Eng. Comm. 2003, 5, 480-486.

19. Mascal, M.; Infantes, L.; Chisholm, J. Water oligomers in crystal hydrates-What's news and what isn't? Angew. Chem.-Int. Ed. 2006, 45, 32-36.

20. Jeffrey, G.A.; Maluszynska, H. The stereochemistry of the water molecules in the hydrates of small biological molecules. Acta Crystallogr. Sect. B 1990, 46, 546-549.

21. Infantes, L.; Fabian, L.; Motherwell, W.D.S. Organic crystal hydrates: what are the important factors for formation. Cryst. Eng. Comm. 2007, 9, 65-71.

22. Haynes, D.A.; Jones, W.; Motherwell, W.D.S. Occurrence of pharmaceutically acceptable anions and cations in the Cambridge structural database. J. Pharm. Sci. 2005, 94, 2111-2120.

23. Haynes, D.A.; Jones, W.; Motherwell, W.D.S. Hydrate formation in NH+-containing salts of pharmaceutically acceptable anions: A CSD survey. Cryst. Eng. Comm. 2005, 7, 342-345.

24. Sansam, B.C.R.; Anderson, K.M.; Steed, J.W. A Simple Strategy for Crystal Engineering Water Clusters. Cryst. Growth Des. 2007, 7, 2649-2653.

25. Hulme, A.T.; Price, S.L. Toward the Prediction of Organic Hydrate Crystal Structures. J. Chem. Theory Comput. 2007, 3, 1597-1608.

26. Morris, K.R. Structural Aspects of Hydrates and Solvates. In Polymorphism in Pharmaceutical Solids; Brittain, H.G., Ed.; Marcel Dekker Ltd: New York, NY, USA, 1999; Chapter 4, pp. 125-181.

27. Potter, B.S.; Palmer, R.A.; Withnall, R.; Chowdhry, B.Z. Aza analogs of nucleic acid bases: infrared and Raman spectra of 5-azauracil and crystal structure of 5-azauracil monohydrate. New J. Chem. 1999, 23, 117-122.

28. Todd, A.M.; Anderson, K.M.; Byrne, P.; Goeta, A.E.; Steed, J.W. Helical or Polar Guest-Dependent Z' $=1.5$ or Z' $=2$ Forms of a Sterically Hindered Bis(urea) Clathrate. Cryst. Growth Des. 2006, 6, 1750-1752.

29. Byrne, P.; Lloyd, G.O.; Clarke, N.; Steed, J.W. A “compartmental” Borromean weave coordination polymer exhibiting saturated hydrogen bonding to anions and water cluster inclusion. Angew. Chemie-Int. Ed. 2008, 47, 5761-5764.

30. Barbour, L.J.; Orr, G.W.; Atwood, J.L. An intermolecular $\left(\mathrm{H}_{2} \mathrm{O}\right)_{10}$ cluster in a solid-state supramolecular complex. Nature 1998, 393, 671-673.

31. Konig, H. A cubic ice modification. Z. Kristallogr. 1944, 105, 279-286.

32. Du, M.; Zhang, Z.-H.; Guo, W.; Fu, X.-J. Multi-Component Hydrogen-Bonding Assembly of a Pharmaceutical Agent Pamoic Acid with Piperazine or 4,4'-Bipyridyl: A Channel Hydrated Salt with Multiple-Helical Motifs vs a Bimolecular Cocrystal. Cryst. Growth Des. 2009, 9, 1655-1657.

33. Gerdil, R. The crystal structure of thymine monohydrate. Acta Crystallogr. 1961, 14, 333-344. 
34. Perrier, P.; Byrn, S.R. Influence of crystal packing on the solid-state desolvation of purine and pyrimidine hydrates: loss of water of crystallization from thymine monohydrate, cytosine monohydrate, 5-nitrouracil monohydrate, and 2'-deoxyadenosine monohydrate. J. Org. Chem. 1982, 47, 4671-4676.

35. Sutor, D.J. The structures of the pyrimidines and purines. VII. The crystal structure of caffeine. Acta Crystallogr. 1958, 11, 453-4588.

36. Edwards, H.G.M.; Lawson, E.; de Matas, M.; Shields, L.; York, P. Metamorphosis of caffeine hydrate and anhydrous caffeine. J. Chem. Soc., Perkin Tran. 1997, 2, 1985-1990.

37. Gerdil, R.; Marsh, R.E. Arrangement of the $\mathrm{H} 2 \mathrm{O}$ molecules in the crystal structure of caffeine. Acta Crystallogr. 1960, 13, 166-167.

38. Sutor, D.J. The structures of the pyrimidines and purines. VI. The crystal structure of theophylline. Acta Crystallogr. 1958, 11, 83-87.

39. Sun, C.; Zhou, D.; Grant, D.J.W.; Young Junior, V.G. Theophylline monohydrate. Acta Crystallogr. Sect. E 2002, 58, o368-o370.

40. Griesser, U.J.; Burger, A. The effect of water vapor pressure on desolvation kinetics of caffeine 4/5-hydrate. Int. J. Pharm. 1995, 120, 83-93.

41. Ahlqvist, M.U.A.; Taylor, L.S. Water dynamics in channel hydrates investigated using H/D exchange. Int. J. Pharm. 2002, 241, 253-261.

42. Suihko, E.; Ketolainen, J.; Poso, A.; Ahlgren, M.; Gynther, J.; Paronen, P. Dehydration of theophylline monohydrate-a two step process. Int. J. Pharm. 1997, 158, 47-55.

43. Kennedy, A.R.; Okoth, M.O.; Sheen, D.B.; Sherwood, J.N.; Teat, S.J.; Vrcelj, R.M. Cephalexin: a channel hydrate. Acta Crystallog. Sect. C 2003, C59, o650-o652.

44. Stephenson, G.A.; Groleau, E.G.; Kleemann, R.L.; Xu, W.; Rigsbee, D.R. Formation of Isomorphic Desolvates: Creating a Molecular Vacuum. J. Pharm. Sci. 1998, 87, 536-542.

45. Saha, B.K.; Nangia, A. First example of an ice-like water hexamer boat tape structure in a supramolecular organic host. Chem. Commun. 2006, 1825-1827.

46. Ringertz, H. Optical and Crystallographic Data of Uric Acid and its Dihydrate. Acta Crystallogr. 1965, 19, 286-287.

47. Artioli, G.; Masciocchi, N.; Galli, E. The elusive crystal structure of uric acid dihydrate: Implication for epitaxial growth during biomineralization. Acta Crystallogr. Sect. B 1997, 53, 498-503.

48. Parkin, S.; Hope, H. Uric acid dihydrate revisited. Acta Crystallogr. Sect. B 1998, 54, 339-344.

49. Bock, H.; Schoedel, H.; Van, T.T.H.; Dienelt, R.; Gluth, M. Interactions in crystals. 136. Protonated dipyridylamine salts with different anions. Monomeric tetraphenylborate as well as bis(trifluoromethylsulfonate), dimeric squarate, and polymeric chloride dihydrate. J. Prakt. Chem./Chem.-Ztg. 1998, 340, 722-732.

50. Ivashevskaya, S.N.; van de Streek, J.; Djanhan, J. E.; Bruening, J.; Alig, E.; Bolte, M.; Schmidt, M.U.; Blaschka, P.; Hoeffken, H.W.; Erk, P. Structure determination of seven phases and solvates of Pigment Yellow 183 and Pigment Yellow 191 from X-ray powder and single-crystal data. Acta Crystallogr. Sect. B 2009, B65, 212-222.

51. Gorbitz, C.H.; Hersleth, H.-P. On the inclusion of solvent molecules in the crystal structures of organic compounds. Acta Crystallogr. Sect. B 2000, B56, 526-534. 
52. Herbst, W.; Hunger, K. Industrial Organic Pigments: Production, Properties, Applications; Wiley-VCH: Weinheim, Germany, 2004.

53. Holleman, A.F. Text Book of Inorganic Chemistry; Veit and Co: Leipzig, Germany, 1906.

54. Oxtoby, N.S.; Blake, A.J.; Champness, N.R.; Wilson, C. Water superstructures within organic arrays; Hydrogen-bonded water sheets, chains and clusters. Chem. Eur. J. 2005, 11, 4643-4654.

55. Vrecer, F.; Vrbinc, M.; Meden, A. Characterization of piroxicam crystal modifications. Int. J. Pharm. 2003, 256, 3-15.

56. Harris, K.D.M. Structure determination of molecular crystals directly from powder diffraction data. Rigaku J. 2001, 18, 23-32.

57. Harris, K.D.M.; Johnston, R.L.; Turner, G.W.; Tedesco, E.; Cheung, E.Y.; Kariuki, B.M. Recent advances in the opportunities for solving molecular crystal structures directly from powder diffraction data. Mol. Cryst. Liq. Cryst. Sci. Technol., Sect. A 2002, 389, 123-129.

58. David, W.I.F.; Shankland, K. Structure determination from powder diffraction data. Acta Crystallogr. Sect. A 2008, A64, 52-64.

59. Redman-Furey, N.; Dicks, M.; Bigalow-Kern, A.; Cambron, R.T.; Lubey, G.; Lester, C.; Vaughn, D. Structural and analytical characterization of three hydrates and an anhydrate form of risedronate. J. Pharm. Sci. 2005, 94, 893-911.

60. Garcia-Garibay, M.A. Molecular crystals on the move: from single-crystal-to-single-crystal photoreactions to molecular machinery. Angew. Chem.-Intl. Ed. 2007, 46, 8945-8947.

61. Das, D.; Engel, E.; Barbour, L.J. Reversible single-crystal to single-crystal polymorphic phase transformation of an organic crystal. Chem. Commun. 2010, 46, 1676-1678.

62. Yin, S.X.; Scaringe, R.P.; Malley, M.F.; Gougoutas, J.Z. In-situ variable temperature powder $\mathrm{X}$-ray diffraction and thermal analysis -applications in the pharmaceutical industry. Am. Pharm. Rev. 2005, 8, 56-58, 60, 62, 67.

63. Tong, H.H.Y.; Chow, A.S.F.; Chan, H.M.; Chow, A.H.L.; Wan, Y.K.Y.; Williams, I.D.; Shek, F.L.Y.; Chan, C.K. Process-induced phase transformation of berberine chloride hydrates. J. Pharm. Sci. 2010, 99, 1942-1954.

64. Zhu, H.J.; Grant, D.J.W. Dehydration behavior of nedocromil magnesium pentahydrate. Int. J. Pharm. 2001, 215, 251-262.

65. Kachrimanis, K.; Fucke, K.; Noisternig, M.; Siebenhaar, B.; Griesser, U.J. Effects of Moisture and Residual Solvent on the Phase Stability of Orthorhombic Paracetamol. Pharm.Res. 2008, 25, 1440-1449.

66. Anderson, K.M.; Day, G.M.; Paterson, M.J.; Byrne, P.; Clarke, N.; Steed, J.W. Structure calculation of an elastic hydrogel from sonication of rigid small molecule components. Angew. Chem.-Intl. Ed. 2008, 47, 1058-1062.

67. Weller, M.T.; Henry, P.F.; Ting, V.P.; Wilson, C.C. Crystallography of hydrogen-containing compounds: realizing the potential of neutron powder diffraction. Chem. Commun. 2009, 2973-2989.

68. Wilson, C.C.; Thomas, L.H. The short hydrogen bond in molecular materials: neutron diffraction and complementary studies. C. R. Chim. 2005, 8, 1434-1443.

69. Adam, M.S.; Gutmann, M.J.; Leech, C.K.; Middlemiss, D.S.; Parkin, A.; Thomas, L.H.; Wilson, C.C. Stability and cooperativity of hydrogen bonds in dihydroxybenzoic acids. New J. Chem. 2010, 34, 85-91. 
70. Thomas, L.H.; Florence, A.J.; Wilson, C.C. Hydrogen atom behaviour imaged in a short intramolecular hydrogen bond using the combined approach of X-ray and neutron diffraction. New J. Chem. 2009, 33, 2486-2490.

71. Wilson, C.C.; Xu, X.; Florence, A.J.; Shankland, N. Temperature dependence of proton transfer in 4-chlorobenzoic acid. New J. Chem. 2006, 30, 979-981.

72. Turner, D.R.; Henry, M.; Wilkinson, C.; McIntyre, G.J.; Mason, S.A.; Goeta, A.E.; Steed, J.W. Cooperative Hydrogen-Bonding Effects in a Water Square: A Single-Crystal Neutron and Partial Atomic Charges and Hardness Analysis Study. J. Am. Chem. Soc. 2005, 127, 11063-11074.

73. Ferraris, G.; Franchini-Angela, M. Survey of the geometry and environment of water molecules in crystalline hydrates studied by neutron diffraction. Acta Crystallogr. Sect. B 1972, 28, 3572-3583.

74. Bouquiere, J.P.; Finney, J.L.; Lehmann, M.S.; Lindley, P.F.; Savage, H.F.J. High-resolution Neutron Study of Vitamin B12 Coenzyme at $15 \mathrm{~K}$ : Structure Analysis and Comparison with thte Structure at 279 K. Acta Crystallogr. Sect. B 1993, 49, 79-89.

75. Bouquiere, J.P.; Finney, J.L.; Savage, H.F.J. High-Resolution Neutron Study of Vitamin B12 Coenzyme at 15 K: Solvent Structure. Acta Crystallogr. Sect. B 1994, 50, 566-578.

76. Bilton, C.; Howard, J.A.K.; Madhavi, N.N.L.; Desiraju, G.R.; Allen, F.H.; Wilson, C.C. Crystal engineering in the gem-alkynol family: the key role of water in the structure of 2,3,5,6-tetrabromo-trans-1,4-diethynyl-cyclohexa-2,5-diene-1,4-diol dihydrate determined by X-ray and neutron diffraction at 150 K. Acta Crystallogr. Sect. B 2001, 57, 560-566.

77. Krebs, F.C.; Jorgensen, M.; Lebech, B.; Frydenvang, K. A perdeuterated cryoprotectant for neutron studies and a demonstration of its use for neutron powder diffraction on L-(-)-ephedrine hemihydrate. J. Appl. Crystallogr. 2001, 34, 203-207.

78. Bordeneuve, H.; Tenailleau, C.; Guillemet-Fritsch, S.; Smith, R.; Suard, E.; Rousset, A. Structural variations and cation distributions in $\mathrm{Mn}_{3-\mathrm{x}} \mathrm{Co}_{\mathrm{x}} \mathrm{O}_{4}(0 \leq \mathrm{x} \leq 3)$ dense ceramics using neutron diffraction data. Solid State Sci. 2010, 12, 379-386.

79. Bull, D.J.; Weidner, E.; Shabalin, I.L.; Telling, M.T.F.; Jewell, C.M.; Gregory, D.H.; Ross, D.K. Pressure-dependent deuterium reaction pathways in the Li-N-D system. Phys. Chem. Chem. Phys. 2010, 12, 2089-2097.

80. Yan, Y.; Telepeni, I.; Yang, S.; Lin, X.; Kockelmann, W.; Dailly, A.; Blake, A.J.; Lewis, W.; Walker, G.S.; Allan, D.R.; Barnett, S.A.; Champness, N.R.; Schroder, M. Metal-Organic Polyhedral Frameworks: High H2 Adsorption Capacities and Neutron Powder Diffraction Studies. J. Am. Chem. Soc. 2010, 132, 4092-4094.

81. Paradowska, A.M.; Price, J.W.H.; Finlayson, T.R.; Lienert, U.; Ibrahim, R. Comparison of Neutron and Synchrotron Diffraction Measurements of Residual Stress in Bead-on-Plate Weldments. J. Pressure Vessel Technol. 2010, 132, 011502/1-011502/8.

82. Paradowska, A.M.; Price, J.W.H.; Kerezsi, B.; Dayawansa, P.; Zhao, X.L. Stress relieving and its effect on life of welded tubular joints. Eng. Failure Anal. 2010, 17, 320-327.

83. Stahly, G.P. Diversity in single- and multiple-component crystals. The search for and prevalence of polymorphs and cocrystals. Cryst. Growth Des. 2007, 7, 1007-1026.

(C) 2010 by the authors; licensee MDPI, Basel, Switzerland. This article is an Open Access article distributed under the terms and conditions of the Creative Commons Attribution license (http://creativecommons.org/licenses/by/3.0/). 